\title{
Prescribed analgesics in pregnancy and risk of childhood asthma
}

\author{
Seif O. Shaheen ${ }^{1}$, Cecilia Lundholm² ${ }^{2}$ Bronwyn K. Brew ${ }^{2}$ and \\ Catarina Almqvist ${ }^{2,3}$
}

\begin{abstract}
Affiliations:
${ }^{1}$ Centre for Primary Care and Public Health, Barts and The London School of Medicine and Dentistry, Queen Mary University of London, London, UK.

${ }^{2}$ Dept of Medical Epidemiology and Biostatistics, Karolinska Institutet, Stockholm, Sweden.

${ }^{3}$ Pediatric Allergy and Pulmonology Unit, Astrid Lindgren Children's Hospital, Karolinska University Hospital, Stockholm, Sweden.
\end{abstract}

\section{Correspondence:}

Seif O. Shaheen, Centre for Primary Care and Public Health, Blizard Institute, Barts and The London School of Medicine and Dentistry, 58 Turner Street, London, E1 2AB, UK.

E-mail: s.shaheendqmul.ac.uk

@ERSpublications

The relationship between prenatal analgesic exposure and childhood asthma is not specific to paracetamol. The association is likely to be confounded by factors intrinsic to the mother, such as chronic pain or anxiety, rather than being causal. http://ow.ly/3LFH30nCS9K

Cite this article as: Shaheen SO, Lundholm C, Brew BK, et al. Prescribed analgesics in pregnancy and risk of childhood asthma. Eur Respir J 2019; 53: 1801090 [https://doi.org/10.1183/13993003.01090-2018].

ABSTRACT Many epidemiological studies have reported a positive association between prenatal exposure to paracetamol and childhood wheezing and asthma. We investigated whether the link between prenatal analgesic exposure and asthma/wheeze is specific to paracetamol, and whether it is causal or confounded.

Using linked Swedish health register data we investigated the relation between various prescribed analgesics in pregnancy and the risk of childhood asthma/wheeze in a population of 492999, and used negative paternal control and sibling comparison approaches to explore unmeasured confounding.

After controlling for potential confounders, prescribed opioids, antimigraine drugs and paracetamol were all positively associated with childhood asthma/wheeze risk at all ages (e.g. for asthma/wheeze at age 4 years: adjusted OR 1.39 (95\% CI 1.30-1.49), 1.19 (95\% CI 1.01-1.40) and 1.47 (95\% CI 1.36-1.59) for opioids, antimigraine drugs and paracetamol, respectively). The results of the paternal control analysis did not suggest the presence of unmeasured confounding by genetics or shared environment. However, the sibling control analysis broadly suggested that associations between prenatal exposure to the analgesics and asthma/wheeze were confounded by specific maternal factors (e.g. for asthma/wheeze at age 4 years: adjusted OR 0.91 (95\% CI 0.62-1.31), 0.50 (95\% CI $0.17-1.45)$ and 0.80 (95\% CI $0.50-1.29$ ) for opioids, antimigraine drugs and paracetamol, respectively).

We propose that analgesic use in pregnancy does not cause childhood asthma/wheeze and that the association is confounded by unmeasured factors that are intrinsic to the mother, such as chronic pain or anxiety. 


\section{Introduction}

Following our original epidemiological observations of a positive association between maternal, self-reported, use of paracetamol (acetaminophen) in pregnancy and risk of pre-school wheezing [1], we subsequently reported a positive association with later childhood asthma [2,3]. A number of other birth cohort studies have confirmed these findings [4], and we originally proposed that, if causal, the associations with prenatal exposure might be explained by increased oxidative stress and depletion of glutathione [1-3]; however, a causal link has not been established. Experimental evidence on effects of prenatal paracetamol in animal models is conflicting $[5,6]$ and a randomised controlled trial to test this hypothesis has not been conducted in humans. Paracetamol is the most commonly used analgesic in pregnancy, so it is important to confirm or refute whether it might be a cause of childhood asthma. Given that a trial in pregnant women would be difficult to undertake [7], there is a need for multiple approaches to rigorously address potential confounding in observational studies.

An important question, relevant to causal inference, is whether the observed epidemiological associations are specific to paracetamol. No association was found with self-reported maternal use of aspirin in pregnancy in our original studies $[1,2]$ nor has there been convincing evidence from other birth cohorts to implicate prenatal exposure to ibuprofen [8-11]. In contrast, a large, population-based study from Denmark using linked national registers reported an increased childhood asthma risk associated with use of prescribed nonsteroidal anti-inflammatory drugs, as well as paracetamol [12]. If the relation between maternal analgesic use in pregnancy and childhood asthma risk is not specific to paracetamol, this would suggest, indirectly, that the association may be confounded by indication, rather than causal, since the various implicated analgesics have different modes of action. One study reported an association between maternal pain in pregnancy and childhood asthma risk, in the absence of paracetamol use [9].

While positive epidemiological associations have remained after controlling for measured confounders using conventional multivariate analysis, the possibility of unmeasured confounding cannot be ruled out. One way to investigate this is to use negative parental controls [13, 14]; if effect estimates for paternal use during pregnancy are similar to those for maternal use during pregnancy, this would suggest confounding by genetic or shared environmental or lifestyle factors, rather than a direct intra-uterine causal effect of prenatal exposure. An alternative family design method, which can be used to assess the possibility of confounding specific to the mother, is sibling control analysis [15]. This approach is based on the principle that siblings share stable aspects of both family environment (prenatal and postnatal) and maternal-specific factors (intra-uterine and behavioural), as well as 50\% of their segregating genes. Sibling control analysis has not been used previously to investigate the relation between prenatal analgesic exposure and asthma.

We have investigated the association between various prescribed analgesics in pregnancy and the risk of childhood asthma/wheeze using Swedish national health and prescription register data, using negative paternal control and sibling comparisons to address unmeasured confounding.

\section{Methods}

\section{Study population}

We identified a cohort of children born to women who became pregnant from July 2005 onwards and gave birth before the end of 2010 from the Medical Birth Register, which includes data on maternal, pregnancy and perinatal factors for $>98 \%$ of all births in Sweden. Data were linked, via individual personal identification numbers assigned to all residents in Sweden, to the Migration Register, the Register of the Total Population, the National Patient Register and the Swedish Prescribed Drug Register (SPDR). Linkage to the Multi-Generation Register allowed identification of the children's fathers and siblings and the Register of the Total Population dates of death. We accessed data from all registers until December 31, 2013. Children who died or emigrated before the age of outcome were excluded (supplementary figure E1).

\section{Exposure}

We collected information on prescribed analgesics dispensed during pregnancy from the SPDR. The nationwide SPDR was introduced in July 2005, and contains information on prescription and dispense dates, number of packages, and dosage of all prescribed medications dispensed in Swedish pharmacies, based on the Anatomical Therapeutic Chemical (ATC) classification system. Drugs of interest were those included in three analgesic classes, i.e. opioids, antimigraine drugs and paracetamol, identified using ATC classification system codes (supplementary table E1). Combined preparations of analgesics across the three classes (e.g. paracetamol plus codeine) were not included, so that we could assess associations with the three classes of analgesics separately. However, a small minority of mothers were prescribed more than one class of analgesic (supplementary table E2). 


\section{Outcome}

Information on childhood asthma was collected from the National Patient Register for all inpatient and specialist outpatient diagnoses (International Classification of Diseases (ICD)-10: J45 and J46), and from the SPDR for all asthma prescribed medications from both primary and specialist care. Prescriptions were used in a previously validated asthma medication algorithm [16]. In brief, the algorithm for current asthma requires at least one asthma prescription in the last 12 months of either: two or more prescriptions for preventer medications (inhaled corticosteroids, leukotriene receptor antagonists, inhaled corticosteroid combinations), or three short-acting $\beta$-agonist prescriptions, or two short-acting $\beta$-agonist and one preventer medication. Children aged $<4.5$ years were required to fulfil both diagnosis and medication criteria in order be more certain that children had asthma rather than pre-school wheeze in this age group. Children aged $>4.5$ years were required to have either a diagnosis or fulfil medication criteria. These registry-based asthma outcomes were objectively assessed, and have previously been validated and found to be specific [16]. Nevertheless, as some "asthma" in those aged $<4.5$ years of age may actually be pre-school wheezing that will not persist as asthma later in childhood, we refer to "asthma/wheeze" as the outcome being analysed. We defined prevalent asthma/wheeze at age 2, 3, 4, 5 and 6 years. As our dataset included children born between 2006 and 2010, and with administrative censoring of outcome data in December 2013, we have fewer children contributing to the analyses of asthma in the older age groups.

\section{Confounders}

We collected information on maternal factors (smoking and body mass index in pregnancy, age at delivery, parity, and country of birth) from the Medical Birth Register. Paternal country of birth and the highest level of education of either parent were obtained from the Longitudinal Integration Database for Health Insurance and Labour Market Studies. Maternal and paternal asthma were defined on the basis of dispensed asthma drugs (data from 2005 onwards) or a diagnosis of asthma ever (ICD-8/9: 493; ICD-10: J45, J46) (data from 2001 onwards).

\section{Ethical approval}

The study was approved by the regional ethics review board in Stockholm, Sweden.

\section{Statistical analysis}

First, we used logistic regression to analyse the association between analgesics prescribed to the mother in pregnancy and prevalent childhood asthma/wheeze at ages 2-6 years in the whole cohort (the same individuals could be included at successive ages in the analyses), controlling for the potential maternal confounders listed earlier. The reference group in all analyses was mothers not prescribed the analgesic being analysed. We analysed prevalent asthma/wheeze separately at each year of age to see whether we observed similar findings for younger children as for older children, given uncertainties over "asthma" versus pre-school wheezing in the younger age groups. We also conducted several sensitivity analyses. 1) In order to increase the likelihood that mothers actually took the prescribed analgesics, we conducted an analysis with analgesic exposure being defined by the drug having been dispensed at least twice during pregnancy. 2) To determine if there was a dose-response effect, we analysed whether increasing the number of classes of drugs increased the strength of the association with asthma/wheeze. 3) In case of increased use of over-the-counter (OTC) prescriptions, or use of earlier prescriptions in subsequent pregnancies (which could attenuate the findings towards the null), we analysed only those children who were first born.

Second, we used logistic regression to analyse the relation between analgesics prescribed to the father during the mother's pregnancy and childhood asthma/wheeze, controlling for potential paternal confounders. We mutually adjusted the paternal analysis for maternal prescription of analgesics in pregnancy and, in turn, mutually adjusted the maternal analysis for paternal prescription of analgesics in pregnancy, because of an observed positive association between maternal and paternal use of analgesics (data not shown).

Third, we used a sibling comparison design with conditional logistic regression. This design utilises the fact that siblings share family environment (prenatal and postnatal) and maternal-specific factors (intra-uterine and behavioural), and adjusts for these unmeasured confounders $[17,18]$. Siblings also share $50 \%$ of the segregating genes. If the association between analgesics and asthma/wheeze in the cohort analysis diminishes, or is attenuated, in the sibling control analysis, it is likely that shared familial factors confound the association. Conversely, if the association remains, then a potential causal explanation is more likely. Controls were all full siblings (excluding children for whom the father's identity was unknown) who did not have asthma and who were still in the study at the age of the particular analysis (2, 3 or 4 years of age). Analogous to a matched case-control study, only siblings who are discordant for both exposure (analgesics prescribed to the mother) and outcome (asthma) are informative for estimation of effect parameters; asthma at 5 and 6 years of age was excluded as there were too few families with 
TABLE 1 Maternal characteristics and asthma/wheeze in the child: overall and by maternal analgesics prescribed in pregnancy

\begin{tabular}{|c|c|c|c|c|}
\hline & All & Opioids & Antimigraine drugs & Paracetamol \\
\hline Subjects & 492999 & 21650 & 3722 & 14732 \\
\hline \multicolumn{5}{|l|}{ Maternal characteristics } \\
\hline \multicolumn{5}{|l|}{ Born in } \\
\hline Sweden & $392886(79.7)$ & 17409 (80.4) & 3212 (86.3) & $9867(67.0)$ \\
\hline Nordics (not Sweden) & $6917(1.4)$ & $336(1.6)$ & 50 (1.3) & $208(1.4)$ \\
\hline EU & $12829(2.6)$ & $431(2.0)$ & $76(2.0)$ & $324(2.2)$ \\
\hline Europe (not EU) & $18718(3.8)$ & 720 (3.3) & 85 (2.3) & $834(5.7)$ \\
\hline Africa & $13475(2.7)$ & $597(2.8)$ & $43(1.2)$ & $697(4.7)$ \\
\hline North America & $1829(0.4)$ & $100(0.5)$ & $7(0.2)$ & $47(0.3)$ \\
\hline South America & $5249(1.1)$ & $295(1.4)$ & $36(1.0)$ & $173(1.2)$ \\
\hline Asia & $40615(8.2)$ & $1737(8.0)$ & $207(5.6)$ & $2571(17.5)$ \\
\hline Oceania & $207(0.0)$ & $12(0.1)$ & $2(0.1)$ & $5(0.0)$ \\
\hline Ex-Soviet Union & $221(0.0)$ & $13(0.1)$ & $4(0.1)$ & $4(0.0)$ \\
\hline Missing & $53(0.0)$ & $0(0.0)$ & $0(0.0)$ & $2(0.0)$ \\
\hline \multicolumn{5}{|l|}{ Education } \\
\hline Elementary school (9 years) & $23792(5.1)$ & $1316(6.1)$ & $125(3.4)$ & $1489(10.1)$ \\
\hline Secondary school (11-12 years) & $181288(36.8)$ & 9469 (43.7) & 1334 (35.8) & $6442(43.7)$ \\
\hline College/university & $286456(58.1)$ & $10827(50.0)$ & $2262(60.8)$ & $6739(45.7)$ \\
\hline Missing & $1463(0.3)$ & $38(0.2)$ & $1(0.0)$ & $62(0.4)$ \\
\hline \multicolumn{5}{|l|}{ Asthma } \\
\hline No & $428771(87.0)$ & $16697(77.1)$ & 2909 (78.2) & 11033 (74.9) \\
\hline Yes & $64228(13.0)$ & 4953 (22.9) & $813(21.8)$ & $3699(25.1)$ \\
\hline \multicolumn{5}{|l|}{$\mathrm{BMI}$ in early pregnancy $\mathrm{kg} \cdot \mathrm{m}^{-2}$} \\
\hline$<18.5$ & $10741(2.2)$ & 403 (1.9) & $58(1.6)$ & $251(1.7)$ \\
\hline $18.5-24.9$ & $275170(55.8)$ & $10112(46.7)$ & $1854(49.8)$ & $6268(42.5)$ \\
\hline $25-29.9$ & $111321(22.6)$ & 5529 (25.5) & $939(25.2)$ & $4013(27.2)$ \\
\hline$\geqslant 30$ & $53990(11.0)$ & $3633(16.8)$ & $542(14.6)$ & $2973(20.2)$ \\
\hline Missing & $41777(8.5)$ & $1973(9.1)$ & $329(8.8)$ & $1227(8.3)$ \\
\hline \multicolumn{5}{|l|}{ Parity } \\
\hline 1 & $216556(43.9)$ & 8223 (38.0) & $1603(43.1)$ & $5112(34.7)$ \\
\hline 2 & $181265(36.8)$ & $7714(35.6)$ & $1254(33.7)$ & $4846(32.9)$ \\
\hline 3 & $66898(13.6)$ & $3678(17.0)$ & $574(15.4)$ & $2707(18.4)$ \\
\hline$\geqslant 4$ & $28280(5.7)$ & $2035(9.4)$ & $291(7.8)$ & $2067(14.3)$ \\
\hline \multicolumn{5}{|l|}{ Smoking during pregnancy } \\
\hline No & $493338(89.1)$ & 18123 (83.7) & 3310 (88.9) & 12507 (84.9) \\
\hline Yes & $32757(6.6)$ & $2561(11.8)$ & $252(6.8)$ & $1664(11.3)$ \\
\hline Missing & $20904(4.2)$ & $966(4.5)$ & $160(4.3)$ & $561(3.8)$ \\
\hline \multicolumn{5}{|l|}{ Maternal age at delivery years } \\
\hline$<20$ & $7760(1.6)$ & $215(1.0)$ & $14(0.4)$ & $149(1.0)$ \\
\hline $20-24$ & $62013(12.6)$ & $2578(11.9)$ & $256(6.9)$ & $1696(11.5)$ \\
\hline $25-29$ & $140780(28.6)$ & $5888(27.2)$ & $833(22.4)$ & $3776(25.6)$ \\
\hline $30-34$ & $173400(35.2)$ & 7466 (34.5) & 1405 (37.7) & $4893(33.2)$ \\
\hline $35-40$ & $90031(18.3)$ & 4445 (20.5) & $965(25.9)$ & $3235(22.0)$ \\
\hline$\geqslant 40$ & 19015 (3.9) & $1058(4.9)$ & $249(6.7)$ & $983(6.7)$ \\
\hline \multicolumn{5}{|l|}{ Childhood asthma/wheeze } \\
\hline At age 2 years $(n=492999)$ & $35708(7.2)$ & 2492 (11.5) & $388(10.4)$ & $1701(11.5)$ \\
\hline At age 3 years $(n=381729)$ & $27876(7.3)$ & $1851(11.4)$ & $305(10.9)$ & $9610(11.9)$ \\
\hline At age 4 years $(n=276333)$ & $19761(7.2)$ & $1220(10.6)$ & $193(9.8)$ & $852(11.1)$ \\
\hline At age 5 years $(n=173131)$ & 11849 (6.8) & $732(10.3)$ & 134 (11.3) & $507(10.8)$ \\
\hline At age 6 years $(n=72778)$ & $4451(6.1)$ & $290(9.5)$ & $51(10.3)$ & $194(9.9)$ \\
\hline
\end{tabular}

Data are presented as $\mathrm{n}$ or $\mathrm{n}(\%)$. EU: European Union; BMI: body mass index.

doubly discordant siblings for asthma at these ages. However, potential confounders (factors that are not shared between siblings and which are unique for each child) were controlled for.

We also analysed the data as time to event outcome (incident asthma) using Cox proportional hazards regression in the full cohort of children with an estimated conception date from July 1, 2005 and onwards, who were born before January 1, 2013 (n>656000). This alternative approach was used to confirm or refute the findings from the logistic regression analyses. Date of onset of asthma was defined as the date of 
the first prescription of asthma medication or diagnosis in the National Patient Register. All children were followed from birth until the earliest of asthma onset, emigration from Sweden, death or December 31, 2013. Attained age was used as the analysis timescale. The proportional hazards assumption was tested based on Schoenfield residuals and found to be violated. Therefore, a model with time-varying effects was applied, with age bands ( $\leqslant 1,>1-2$ and $>2$ years) decided based on the data. Results are presented as hazard ratios within each age band with corresponding $95 \%$ confidence intervals.

All analyses were performed using Stata version 13 (StataCorp, College Station, TX, USA).

\section{Results}

Table 1 shows maternal characteristics and childhood asthma/wheeze at different ages according to maternal analgesics prescribed in pregnancy. Overall, during pregnancy, $7 \%$ of women had a prescription for an analgesic: $4.4 \%$ were prescribed opioids, $0.8 \%$ were prescribed antimigraine drugs and $3.1 \%$ were prescribed paracetamol. The prevalence of childhood asthma/wheeze was 6-7\%. After controlling for potential confounders, prescribed opioids, antimigraine drugs and paracetamol were all positively associated with childhood asthma/wheeze risk at all ages (figure 1 and table 2; unadjusted results shown in supplementary table E3), e.g. for asthma/wheeze at age 4 years: adjusted OR 1.39 (95\% CI 1.30-1.49), 1.19 (95\% CI 1.01-1.40) and 1.47 (95\% CI 1.36-1.59) for opioids, antimigraine drugs and paracetamol, respectively. Independent associations with each analgesic remained, although attenuated, on mutual adjustment for the other analgesics (supplementary table E4). When we repeated the analyses shown in table 2, with exposure being defined by the drug having been dispensed at least twice during pregnancy, the effect estimates increased in magnitude (supplementary table E5). With an increasing number of classes of analgesics prescribed to women during pregnancy, the strength of the associations with asthma at ages 2, 3 and 5 years increased (supplementary table E6). Including only first-born children in the analysis did not change effect estimates (supplementary table E7).

When we analysed the associations between analgesics prescribed to the father and childhood asthma/ wheeze, all associations were null (table 3 and figure 1), e.g. for asthma/wheeze at age 4 years: adjusted OR 1.01 (95\% CI 0.93-1.09), 1.12 (95\% CI 0.92-1.37) and 1.03 (95\% CI 0.94-1.13) for opioids, antimigraine drugs and paracetamol, respectively. Mutually adjusting maternal analyses for paternal use of analgesics during the mother's pregnancy did not change the maternal effect estimates (data not shown). In the sibling control analysis, none of the analgesics prescribed to the mother in pregnancy were associated with childhood asthma/wheeze at ages 2, 3 and 4 years, e.g. for asthma/wheeze at age 4 years: adjusted OR 0.91

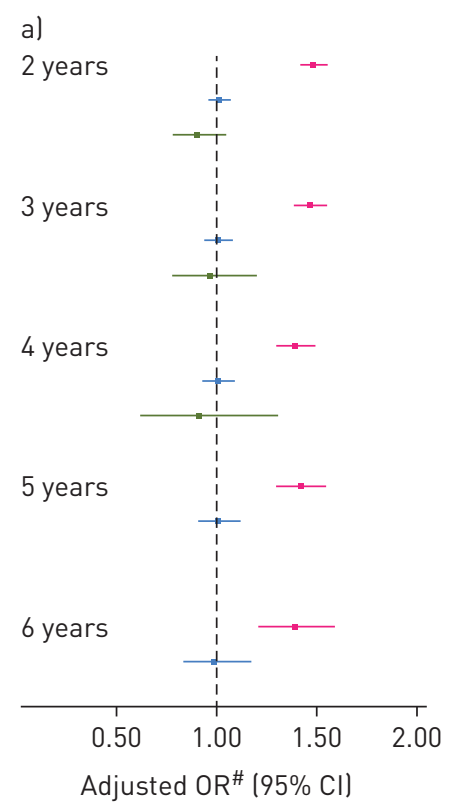

Whole cohort analysis b)

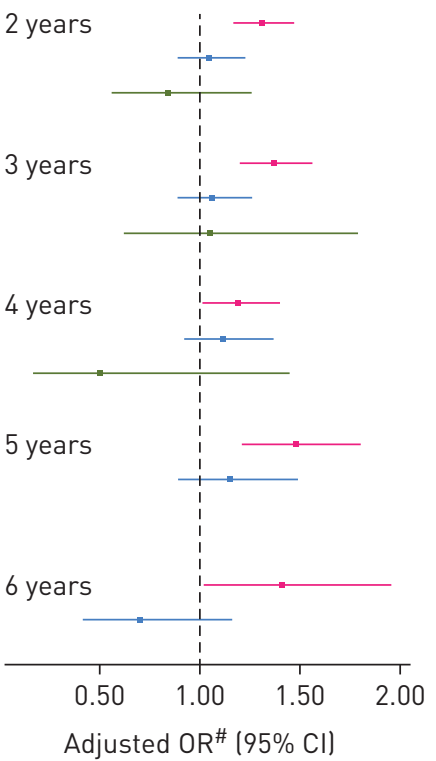

Negative control analysis

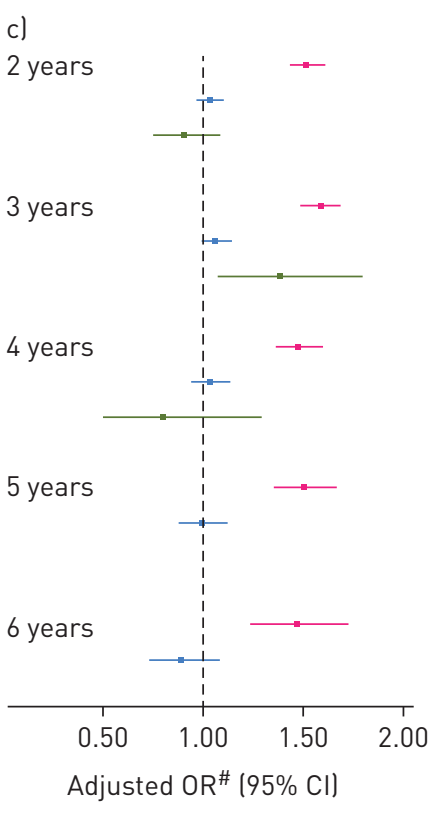

Sibling control analysis

FIGURE 1 Associations between prescribed analgesics in pregnancy and childhood asthma/wheeze: maternal analgesic use (whole cohort analysis; at age 2-6 years), paternal analgesic use (negative control analysis; at age 2-6 years) and maternal analgesic use (sibling control analysis; at age 2-4 years). a) Opioids, b) antimigraine drugs and c) paracetamol. \# : adjusted OR (see tables 2-4). 
TABLE 2 Associations between analgesics prescribed to the mother in pregnancy and childhood asthma/wheeze (whole cohort analysis)

\begin{tabular}{lcccr}
$\begin{array}{c}\text { Asthma/wheeze at } \\
\text { age years }\end{array}$ & Subjects $\mathbf{n}$ & Opioids & Antimigraine drugs & Paracetamol \\
\hline $\mathbf{2}$ & 449934 & $1.48(1.42-1.55)$ & $1.31(1.17-1.47)$ & $1.51(1.43-1.60)$ \\
$\mathbf{3}$ & 346223 & $1.47(1.39-1.55)$ & $1.37(1.20-1.56)$ & $1.58(1.48-1.68)$ \\
$\mathbf{4}$ & 248785 & $1.39(1.30-1.49)$ & $1.19(1.01-1.40)$ & $1.47(1.36-1.59)$ \\
$\mathbf{5}$ & 152797 & $1.42(1.30-1.55)$ & $1.48(1.21-1.80)$ & $1.50(1.35-1.66)$ \\
$\mathbf{6}$ & 64642 & $1.39(1.21-1.59)$ & $1.41(1.02-1.96)$ & $1.46(1.23-1.72)$
\end{tabular}

Associations are presented as adjusted OR $(95 \% \mathrm{Cl})$; adjusted for maternal birth country, education, asthma, body mass index in early pregnancy, parity, smoking during pregnancy and age at delivery.

(95\% CI 0.62-1.31), 0.50 (95\% CI 0.17-1.45) and 0.80 (95\% CI 0.50-1.29) for opioids, antimigraine drugs and paracetamol, respectively, with the exception of a positive association between paracetamol and asthma/wheeze at age 3 years (table 4 and figure 1). This latter association $(p=0.014)$ was no longer conventionally significant after Bonferroni correction.

When we used Cox proportional hazards regression the results were in keeping with those of the logistic regression results presented in tables 2 and 4. In the whole cohort, positive associations with asthma/ wheeze were seen with asthma/wheeze incidence for all three analgesic classes (supplementary table E8a); in contrast, in the sibling control analysis, no associations were seen for any of the analgesics (supplementary table E8b).

\section{Discussion}

In this large population-based study we found that, after controlling for measured confounders, various analgesics prescribed in pregnancy, i.e. opioids, antimigraine drugs and paracetamol, were associated with an increased risk of childhood asthma/wheeze. These associations were stronger when the analgesics had been dispensed twice or more during pregnancy. While the findings for paracetamol, including prescribed paracetamol, are not new $[4,12]$, to the best of our knowledge only one previous study has suggested that prescribed opioids may also be associated with childhood asthma risk [11]; that study found an association with prescribed antimigraine drugs in pregnancy of borderline significance. Our findings were consistent whether we analysed prevalent or incident asthma/wheeze.

\section{Investigation of confounding}

We used two approaches to investigate whether the associations between prenatal exposure to analgesics and childhood asthma/wheeze might be explained by unmeasured confounding. First, we used a negative paternal control approach. We found no association between paternal analgesic use and childhood asthma/wheeze, which is in keeping with two previous studies $[9,19]$, and suggests that the maternal analgesic-asthma/wheeze associations were not confounded by unmeasured genetic, environmental, socioeconomic or lifestyle factors shared by the mother and father, which might influence both the

TABLE 3 Associations between paternal analgesic prescription during mother's pregnancy and childhood asthma/wheeze (negative control analysis)

\begin{tabular}{lcccr}
$\begin{array}{c}\text { Asthma/wheeze at } \\
\text { age years }\end{array}$ & Subjects $\mathbf{n}$ & Opioids & Antimigraine drugs & Paracetamol \\
\hline $\mathbf{2}$ & 445726 & $1.01(0.96-1.07)$ & $1.05(0.89-1.23)$ & $1.03(0.97-1.10)$ \\
$\mathbf{3}$ & 348335 & $1.01(0.94-1.08)$ & $1.06(0.89-1.26)$ & $1.06(0.99-1.14)$ \\
$\mathbf{4}$ & 246912 & $1.01(0.93-1.09)$ & $1.12(0.92-1.37)$ & $1.03(0.94-1.13)$ \\
$\mathbf{5}$ & 152797 & $1.01(0.91-1.12)$ & $1.15(0.89-1.49)$ & $0.99(0.88-1.12)$ \\
$\mathbf{6}$ & 64229 & $0.99(0.84-1.17)$ & $0.70(0.42-1.16)$ & $0.89(0.73-1.08)$ \\
\hline
\end{tabular}

Associations are presented as adjusted OR (95\% Cl); adjusted for paternal birth country, education, paternal asthma, parity, maternal asthma, maternal birth country, body mass index in early pregnancy, smoking during pregnancy, maternal age at delivery and maternal analgesic prescription during pregnancy. 
TABLE 4 Associations between analgesics prescribed to the mother in pregnancy and childhood asthma/wheeze (sibling control analysis)

\begin{tabular}{lcccr}
$\begin{array}{l}\text { Asthma/wheeze } \\
\text { at age years }\end{array}$ & Subjects $\mathbf{n}^{\#}$ & Opioids & Antimigraine drugs & Paracetamol \\
\hline $\mathbf{2}$ & 17522 & $0.90(0.78-1.05)$ & $0.84(0.56-1.26)$ & $0.90(0.75-1.08)$ \\
$\mathbf{3}$ & 9456 & $0.97(0.78-1.20)$ & $1.05(0.62-1.79)$ & $1.38(1.07-1.79)$ \\
$\mathbf{4}$ & 3764 & $0.91(0.62-1.31)$ & $0.50(0.17-1.45)$ & $0.80(0.50-1.29)$ \\
\hline
\end{tabular}

Associations are presented as adjusted OR $(95 \% \mathrm{Cl})$; adjusted for maternal body mass index in early pregnancy, parity, smoking during pregnancy and age at delivery. \#: number of children in families with siblings doubly discordant for asthma and exposures.

propensity for taking prescribed analgesics and the risk of asthma in the offspring. However, we also used a sibling comparison approach, which to the best of our knowledge has not been used previously to test this hypothesis. This showed that the three classes of analgesic were not associated with asthma/wheeze at 2, 3 and 4 years, with one exception: paracetamol was associated with asthma/wheeze at 3 years; while the latter association may be a chance finding, we cannot rule out a causal association. However, the predominant lack of association across the sibling control analyses (which was corroborated when we analysed incident asthma/wheeze using Cox regression) suggests that a large causal effect of maternal analgesic use on risk of childhood asthma/wheeze is unlikely, since the sibling exposed to the maternal use of analgesics had no higher risk of the asthma/wheeze outcomes than the unexposed sibling (although we acknowledge that, given the wide confidence limits, a small/modest effect cannot be ruled out). This suggests that, although the paternal negative control analysis ruled out confounding by unmeasured genetic factors or shared environment, the sibling control analysis indicated possible confounding of the associations between prenatal analgesic exposure and asthma/wheeze by unmeasured factors, which are intrinsic to the mother, and may be common to successive pregnancies. We speculate that these might include a physiological condition such as chronic pain or anxiety, or a behavioural trait such as a propensity to seek healthcare. Confirmation that the relation between prenatal analgesic exposure and asthma/wheeze was not restricted to paracetamol also supports confounding by a stable factor intrinsic to the mother as a likely explanation, given that the three types of analgesic have different mechanisms of action. With regard to chronic pain as a possible maternal confounder, a recent birth cohort study found some evidence that maternal pain, in the absence of paracetamol use, was associated with an increased risk of childhood asthma [9]. Anxiety has been linked to increased prescribed opioid use in pregnancy [20] and anxiety can exacerbate pain sensation [21]. Maternal psychological stress, in turn, has been linked to childhood asthma risk [22], so could potentially be a confounder, although previous studies have shown that the association between prenatal paracetamol exposure and asthma remains after adjusting for maternal anxiety [1-3].

\section{Strengths and limitations}

Aside from its large size, our study has a number of important strengths. First, the linkage of national registers using objectively and prospectively collected measures of exposure and validated outcomes [16], which enables virtually complete coverage and follow-up of the Swedish population, thus reducing the risk of selection and recall bias that can arise in birth cohort studies. Second, the use of negative control analysis and the novel use of sibling control analysis to address unmeasured familial confounding. Third, whether we analysed prevalent asthma/wheeze using logistic regression or incident asthma/wheeze using Cox proportional hazards regression, the results for the whole cohort and in the sibling control analyses were similar.

A limitation of our dataset is that we had fewer children contributing to the analyses of asthma in the older age groups, especially in the sibling control analysis. A future study, designed to capture a larger number of children with asthma at older ages, could be informative. Regarding other potential limitations, while we analysed prescribed paracetamol, this drug is also readily obtained OTC, which could potentially lead to misclassification of exposure to this drug. However, in Sweden, paracetamol is only available OTC in smaller doses and package sizes for a relative increased cost, and therefore it is more likely to be used sporadically rather than for long-term use; individuals needing frequent use tend to get the drug prescribed as this is cheaper. Furthermore, any exposure misclassification is likely to be random with respect to childhood asthma/wheeze and this would be expected to lead to underestimation of effect estimates (as would any exposure misclassification arising from mothers not actually taking analgesics that they had been prescribed, although we at least know that the prescribed drugs were dispensed). In contrast, 
opioids (e.g. codeine, dextropropoxyphene and tramadol) and antimigraine drugs cannot be obtained OTC in Sweden, and therefore the results for these analgesics could be regarded as more convincing, as exposure misclassification will be minimal. Reduced exposure misclassification might also explain why we observed stronger associations when exposure was defined by the drug having been dispensed at least twice in pregnancy, as we can be more confident that exposed mothers actually took the drug during pregnancy. Given the likely sporadic use of OTC paracetamol, we think it unlikely that minor usage of OTC paracetamol will have confounded the associations seen with opioids and antimigraine drugs.

While a proportion of children aged $<5$ years with a hospital diagnosis of asthma and receiving asthma medication may have had pre-school wheezing, our analyses are still valid, as previous epidemiological studies have linked prenatal paracetamol exposure to early childhood wheezing [1], as well as to later asthma $[2,3]$.

\section{Conclusions}

In summary, our findings confirm that analgesic use in pregnancy is associated with an increased risk of childhood asthma/wheeze, but this link is not specific to paracetamol and is unlikely to be causal. We posit that factors intrinsic to the mother and common across pregnancies, such as chronic pain or anxiety, may be confounding the prenatal analgesic-asthma/wheeze association. There have been previous calls for placebo-controlled trials to definitively test the paracetamol-asthma hypothesis [23]. We would argue, on the basis of our latest findings, that the case for a trial in pregnancy, which would present considerable practical and ethical challenges, is now less strong.

Acknowledgements: We thank Christina Norrby and Marcus Boman (Karolinska Institutet, Stockholm, Sweden) for their excellent data collection and management, and Anne Ortqvist (Karolinska Institutet) for helpful discussions.

Author contributions: S.O. Shaheen and C. Almqvist conceived the project. C. Lundholm and B.K. Brew carried out the statistical analyses. S.O. Shaheen drafted the manuscript and all authors contributed to, and approved, the final version.

Conflict of interest: C. Lundholm has nothing to disclose. B.K. Brew has nothing to disclose. C. Almqvist reports grants from the Swedish Research Council through the Swedish Initiative for Research on Microdata in the Social and Medical Sciences (SIMSAM) framework (grant number 340-2013-5867), Stockholm County Council (ALF projects), Swedish Heart-Lung Foundation, FORTE (grant number 2015-00289), and the Swedish Asthma and Allergy Association's Research Foundation, during the conduct of the study. S.O. Shaheen has nothing to disclose.

Support statement: This work was supported by Forskningsrådet om Hälsa, Arbetsliv och Välfärd (FORTE: grant 2015-00289), Hjärt-Lungfonden, Stockholms Läns Landsting (ALF projects), the Swedish Asthma and Allergy Association's Research Foundation and the Swedish Initiative for Research on Microdata in the Social and Medical Sciences (SIMSAM) framework (grant 340-2013-5867). B.K. Brew is supported by FORTE and the European Commission under a COFAS Marie Curie Fellowship. Funding information for this article has been deposited with the Crossref Funder Registry.

\section{References}

1 Shaheen SO, Newson RB, Sherriff A, et al. Paracetamol use in pregnancy and wheezing in early childhood. Thorax 2002; 57: 958-963.

2 Shaheen SO, Newson RB, Henderson AJ, et al. Prenatal paracetamol exposure and risk of asthma and elevated immunoglobulin E in childhood. Clin Exp Allergy 2005; 35: 18-25.

3 Shaheen SO, Newson RB, Ring SM, et al. Prenatal and infant acetaminophen exposure, antioxidant gene polymorphisms, and childhood asthma. J Allergy Clin Immunol 2010; 126: 1141-1148.

4 Fan G, Wang B, Liu C, et al. Prenatal paracetamol use and asthma in childhood: a systematic review and meta-analysis. Allergol Immunopathol 2017; 45: 528-533.

5 Lee DCP, Walker SA, Byrne AJ, et al. Perinatal paracetamol exposure in mice does not affect the development of allergic airways disease in early life. Thorax 2015; 70: 528-536.

6 Karimi K, Keßler T, Thiele K, et al. Prenatal acetaminophen induces liver toxicity in dams, reduces fetal liver stem cells, and increases airway inflammation in adult offspring. J Hepatol 2015; 62: 1085-1091.

7 Cheelo M, Lodge CJ, Dharmage SC, et al. Paracetamol exposure in pregnancy and early childhood and development of childhood asthma: a systematic review and meta-analysis. Arch Dis Child 2015; 100: 81-89.

8 Sordillo JE, Scirica CV, Rifas-Shiman SL, et al. Prenatal and infant exposure to acetaminophen and ibuprofen and the risk for wheeze and asthma in children. J Allergy Clin Immunol 2015; 135: 441-448.

9 Magnus MC, Karlstad O, Haberg SE, et al. Prenatal and infant paracetamol exposure and development of asthma: the Norwegian Mother and Child Cohort Study. Int J Epidemiol 2016; 45: 512-522.

10 Rebordosa C, Kogevinas M, Sorensen HT, et al. Prenatal exposure to paracetamol and risk of wheezing and asthma in children: a birth cohort study. Int J Epidemiol 2008; 37: 583-590.

11 Kallen B, Finnstrom O, Nygren KG, et al. Maternal drug use during pregnancy and asthma risk among children. Pediatr Allergy Immunol 2013; 24: 28-32.

12 Andersen $\mathrm{AB}$, Farkas DK, Mehnert F, et al. Use of prescription paracetamol during pregnancy and risk of asthma in children: a population-based Danish cohort study. Clin Epidemiol 2012; 4: 33-40.

13 Brew BK, Gong T, Williams DM, et al. Using fathers as a negative control exposure to test the Developmental Origins of Health and Disease Hypothesis: a case study on maternal distress and offspring asthma using Swedish register data. Scand J Public Health 2017; 45: 36-40. 
14 Richmond RC, Al-Amin A, Davey Smith G, et al. Approaches for drawing causal inferences from epidemiological birth cohorts: a review. Early Hum Dev 2014; 90: 769-780.

15 Ortqvist $\mathrm{AK}$, Lundholm $\mathrm{C}$, Kieler $\mathrm{H}$, et al. Antibiotics in fetal and early life and subsequent childhood asthma: nationwide population based study with sibling analysis. BMJ 2014; 349: g6979.

16 Ortqvist AK, Lundholm C, Wettermark B, et al. Validation of asthma and eczema in population-based Swedish drug and patient registers. Pharmacoepidemiol Drug Saf 2013; 22: 850-860.

17 D'Onofrio BM, Lahey BB, Turkheimer E, et al. Critical need for family-based, quasi-experimental designs in integrating genetic and social science research. Am J Public Health 2013; 103: Suppl. 1, S46-S55.

18 D’Onofrio BM, Class QA, Rickert ME, et al. Translational epidemiologic approaches to understanding the consequences of early-life exposures. Behav Genet 2016; 46: 315-328.

19 Shaheen SO, Newson RB, Davey Smith G, et al. Prenatal paracetamol exposure and asthma: further evidence against confounding. Int J Epidemiol 2010; 39: 790-794.

20 Smith MV, Costello D, Yonkers KA. Clinical correlates of prescription opioid analgesic use in pregnancy. Matern Child Health J 2015; 19: 548-556.

21 Ploghaus A, Narain C, Beckmann CF, et al. Exacerbation of pain by anxiety is associated with activity in a hippocampal network. J Neurosci 2001; 21: 9896.

22 Flanigan C, Sheikh A, DunnGalvin A, et al. Prenatal maternal psychosocial stress and offspring's asthma and allergic disease: a systematic review and meta-analysis. Clin Exp Allergy 2018; 48: 403-414.

23 Holgate ST. The acetaminophen enigma in asthma. Am J Respir Crit Care Med 2011; 183: 147-151. 